\title{
Infrared Spectroscopy of Light Impurities in $\mathrm{GaSb}$
}

\author{
P. KaCzor, Z. Kaliński And R. JakięA \\ Institute of Physics, Polish Academy of Sciences \\ al. Lotników 32/46, 02-668 Warszawa, Poland
}

\begin{abstract}
In our work we study the doping behaviour of sulphur in Czochralski grown GaSb by means of the high resolution Fourier transform infrared spectroscopy and the secondary ion mass spectroscopy. We have revealed that the sulphur impurity forms an effective mass like donor state bound to the $L$-minimum of the conduction band. From the far infrared spectrum of this donor we derive the effective band masses of the $L$ band minimum. We also observe local vibrational modes related to the arsenic and phosphorus isoelectronic impurities. From the nearest neighbour isotope splittings of these modes we conclude that the arsenic impurity occupies a tetrahedral substitutional site and the phosphorus impurity - a low symmetry lattice site.
\end{abstract}

PACS numbers: 71.55.Eq, 63.20.Pw

\section{Introduction}

Unique properties of GaSb and its alloys such as band structure (spin-orbit splitting energy almost equal to the band gap energy, subsidiary band minimum $L$ only $80 \mathrm{meV}$ above the $\Gamma$ minimum) and doping behaviour ( $p$-type conductivity due to native defects, anomalously low self-diffusion on the antimony site) have attracted interest of basic [1] and applied [2] research. Although the CurieWeiss critical temperature for GaMnSb is low $(\sim 25 \mathrm{~K})$ the compound has been successfully applied for testing various applications of the spintronic devices [3].

Despite great research effort in the recent years many properties of $\mathrm{GaSb}$ are still not well known in detail as compared to other III-V compounds. One of the major issues remaining unsolved is that of the nature of the intrinsic native defects and their interaction with various extrinsic dopants [2].

In our study we use sulphur impurity as a dopant for two reasons: (1) it is a light impurity - we expect local vibrational modes (LVMs) and gap modes 
to appear either due to isolated sulphur on a tetrahedral substitutional site or due to its complexes with native defects; (2) its doping behaviour is very peculiar (it forms a deep, so-called DX-center) and we would like to elucidate it.

\section{Crystal growth and experimental techniques}

The samples used in our investigation were $5-10 \mathrm{~mm}$ diameter slices cut from bulk crystals grown by means of the Czochralski growth with growth parameters changed in order to obtain GaSb:S crystals with various doping levels and conductivity types.

For the $p$-type samples the carrier concentration (as estimated by room temperature Hall effect measurements) ranged from $p=1 \times 10^{15} \mathrm{~cm}^{-3}$ to $2 \times 10^{17} \mathrm{~cm}^{-3}$ (undoped samples, not used in the absorption measurement) at room temperature. We obtained also $n$-type samples with carrier concentrations depending on the sulphur content and varying from $n=2 \times 10^{15} \mathrm{~cm}^{-3}$ to $n=2 \times 10^{16} \mathrm{~cm}^{-3}$. All $n$-type samples exhibited the persistent photoconductivity effect (PPC-effect) at low temperatures.

For the employed by us Czochralski growth technique the native acceptor concentration in GaSb is of the order of $1 \times 10^{17} \mathrm{~cm}^{-3}$ [2], thus we can assume the compensation ratio (i.e. the ratio of the amount of the majority dopants to the minority ones) for the samples ( $p$ - as well as $n$-type) used in the absorption measurement to be of the order of $0.8-0.9$.

With our experimental techniques of choice, the Fourier transform infrared spectroscopy (FTIRS) and the secondary ion mass spectroscopy (SIMS), we were able to monitor in great detail the doping with sulphur and also the residual dopant content in our samples.

The high resolving power of our BOMEM DA3 spectrometer being of the order of $0.1 \mathrm{~cm}^{-1}$ its sensitivity and the relatively broad range of the absorption measurement $\left(80-600 \mathrm{~cm}^{-1}\right)$ allowed us to detect the details either of the LVM absorption (so-called nearest neighbour isotope splitting) as well as of the absorption due to electronic transitions. The use of the variable temperature continuous flow cryostat (5-300 K) allowed us to study the temperature behaviour of the spectral features which helped to discriminate between the electronic or vibrational character of the transitions. Varying the thickness of the samples (typically 0.2 and $0.8 \mathrm{~mm}$ ) we were allowed to vary the relative contribution of the lattice and the residual free carrier absorption to the total absorption coefficient.

With the SIMS technique alone we were not able to estimate the absolute content of impurities in our samples (lack of implantation sources for calibration) but the accuracy of the relative measurement was high enough to allow a detailed comparison of the impurity content between samples. To have the idea of a typical impurity contamination in GaSb we measured also standard substrate samples of undoped and tellurium doped GaSb. 


\section{Results}

The absorption spectrum relevant to impurities present in our GaSb samples is presented in Fig. 1. The dominant absorption features in all $n$-type samples are transitions from the ground state of the effective mass like donor to its excited states. These transitions are not present in $p$-type samples regardless of the sulphur content and scale with $\mathrm{S}$ content for $n$-type samples. They weaken appreciably at temperatures starting from $40 \mathrm{~K}$ and disappear completely at approximately $80 \mathrm{~K}\left(1 s-2 p_{0}\right.$ transition) and $150 \mathrm{~K}\left(1 s-2 p_{ \pm}\right.$transitions $)$suggesting activated behaviour. The transitions could not be directly correlated with the PPC effect observed in the $n$-type samples.

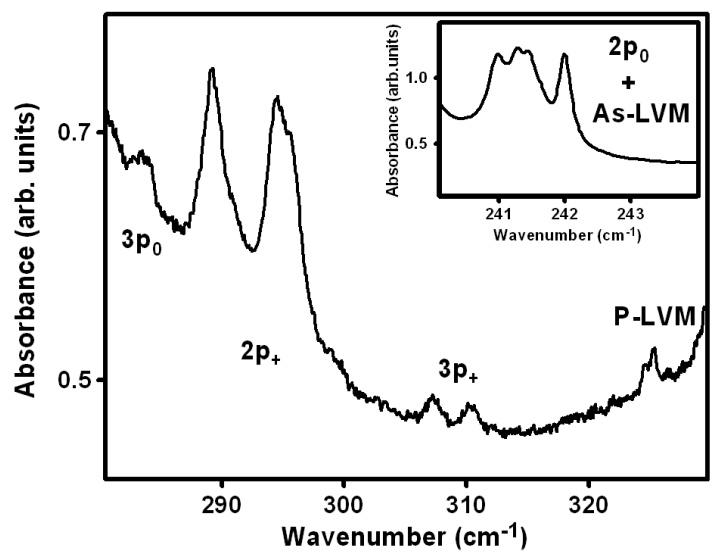

Fig. 1. The infrared spectrum of the $L$-minimum bound donor in GaSb:S and of the LVM due to residual phosphorus and arsenic measured at $5 \mathrm{~K}$ for the sample with $n=10^{16} \mathrm{~cm}^{-3}$. The electronic transitions are labelled by the excited states to which they take place. In the inset the As impurity LVM ( 5 lines due to isotope splitting) is shown overlapping with the $1 s-2 p_{0}$ transition centred at $241.3 \mathrm{~cm}^{-1}$. The local vibrational modes due to residual impurities are absent in the samples containing no traceable amount of As and P as measured by SIMS. The background in the spectra is due to host lattice absorption.

The observed spectral features resemble strongly the infrared spectrum of the antimony donor in Ge [4]. The relatively large linewidth $\left(\sim 1 \mathrm{~cm}^{-1}\right)$ of the transition lines is typical of shallow donor absorption in highly doped and/or compensated semiconductor [5]. The whole spectrum can be explained with a model based on the effective mass approximation for a donor "tied" to a prolate-spheroid conduction band minimum. The binding energies of the excited states are then derived from the paper of Faulkner [6]. Excellent agreement is obtained assuming valley-orbit splitting of the donor ground state of the order of $0.61 \mathrm{meV}$, the ratio of the transversal effective mass to the longitudinal effective mass $\gamma=0.1$ and the 
value of the transversal mass $m_{\mathrm{t}}=0.275 \mathrm{~m}_{0}$. The values of the mass and mass ratio are almost exactly the ones obtained for the $L$-minimum of the conduction band of GaSb from Shubnikov-de Haas measurement [7]. Each transition to the excited state is a doublet (valley-orbit splitting of the $1 s$ state into $A_{1}$ and $E$ states) which additionally supports our model. The binding energy of the donor ground state estimated from the calculated position of the photoionization threshold [4] is $41.5 \mathrm{meV}$. The observed temperature dependence of the strength of transition lines supports this estimation.

It is commonly accepted that the lowest conduction band minimum in $\mathrm{GaSb}$ is the $\Gamma$ minimum. The shallow donor in GaSb:S should therefore have the binding energy of the order of $2 \mathrm{meV}$. Nevertheless the donor state observed in our experiment exhibits features of a state "tied" to a subsidiary minimum with a binding energy an order of magnitude greater.

The other spectral features observed in our GaSb:S samples we attribute to the local vibrational modes of residual impurities. The LVM line located at $325 \mathrm{~cm}^{-1}$ (P-LVM label in Fig. 1) is due to phosphorus involving complex of low symmetry, although the position of the line almost exactly matches the one found by Hayes [8] and assigned by him to the tetrahedral substitutional P impurity. The revealed nearest neighbour fine structure of this line does not support this assignment. Another LVM located at ca. $241.5 \mathrm{~cm}^{-1}$ with nearest neighbour fine structure consisting of five lines characteristic of tetrahedral site substitution is due to As impurity replacing Sb. The lines due to P and As LVM can be observed only in samples with measurable amounts (as measured by SIMS) of the As and $\mathrm{P}$ impurities.

Contrary to our expectations we did not observe any LVM absorption which could be related to sulphur impurity and/or its complexes. This somewhat disappointing result is not so surprising in view of the fact that despite the efforts undertaken in the past 30 years to find the sulphur impurity related LVMs in various III-V compounds the only vibrational modes which can be related to sulphur are the gap modes in GaP [9]. The most probable reason for the lack of the LVM spectra in III-Vs is the extreme weakness of the nearest neighbour force constants [9].

\section{Conclusions}

In our study we attempted to observe the LVM of sulphur in GaSb. No LVM lines observed could be attributed to sulphur vibrations. Instead we discovered transitions from the subsidiary minimum ( $L$-minimum) "bound" donor states in a direct gap semiconductor in which the $\Gamma$ minimum is nominally the lowest conduction band minimum. We also found the nearest neighbour isotope splittings of the $\mathrm{P}$ and As impurities in GaSb. 


\section{References}

[1] H. Bracht, S.P. Nicols, W. Walukiewicz, J.P. Silveira, F. Briones, E.E. Haller, Nature 408, 69 (2000).

[2] P.S. Dutta, H.L. Bhat, V. Kumar, J. Appl. Phys. 81, 5821 (1997).

[3] I. Vurgraftman, R.J. Mayer, Phys. Rev. B 67, 125209 (2003).

[4] J.H. Reuszer, P. Fisher, Phys. Rev. 165, 909 (1968).

[5] Y. Nisida, K. Horii, J. Phys. Soc. Jpn. 26, 388 (1969).

[6] R.A. Faulkner, Phys. Rev. 184, 713 (1969).

[7] P. Perrier, J.C. Portal, J. Galibert, S. Askenazy J. Phys. Soc. Jpn. 49, Suppl. A, 113 (1980).

[8] W. Hayes, Phys. Rev. Lett. 13, 275 (1964).

[9] R.C. Newman, in: Semiconductors and Semimetals, Eds. R.K. Willardson, A.C. Beer, E.R. Weber, Vol. 38, Academic Press, Boston 1998; p. 117; R.S. Leigh, M.J.L. Sangster, R.C. Newman, J.P. Goss, R. Jones, V.J.B. Torres, S. Oeberg, P.R. Briddon, Phys. Rev. B 68, 033304 (2003). 